\title{
Perfil de dores lombares em Fisioterapeutas que atuam em clínicas ambulatoriais em
}

\section{Santarém-PA}

\author{
Back pain profile in physiotherapists who key work in outpatient clinics in Santarém-PA \\ Perfil de dolor de espalda em fisioterapeuta que trabajan en clínicas ambulatorias de Santarém-PA
}

Recebido: 01/10/2021 | Revisado: 07/10/2021 | Aceito: 08/10/2021 | Publicado: 10/10/2021

Eliabe Aguiar da Costa ORCID: https://orcid.org/0000-0002-3529-223X Centro Universitário da Amazônia, Brasil E-mail: eliabeaguiar@gmail.com

Tiago Ribeiro de Sousa ORCID: https://orcid.org/0000-0001-7532-1712 Centro Universitário da Amazônia, Brasil E-mail: tiagofisio2018@gmail.com Assis Júnior Cardoso Pantoja ORCID: https://orcid.org/0000-0002-8301-3135 Centro Universitário da Amazônia, Brasil E-mail: https://assisfisiojr_hotmail.com/

Daliane Ferreira Marinho

ORCID: https://orcid.org/0000-0003-3849-1215 Universidade do Estado do Pará, Brasil E-mail: dalianefmarinho@gmail.com

\begin{abstract}
Resumo
A lombalgia é uma patologia que afeta diversos grupos de trabalhadores em âmbito mundial, sendo uma das afecções musculoesqueléticas mais comuns atualmente. Ela pode interferir na biomecânica de todo o segmento da coluna, levando a restrição de movimento e diminuição da força muscular, o que reflete em afastamentos da atividade laboral e, consequentemente, na qualidade de vida desses indivíduos. Esse estudo buscou analisar o perfil da incidência de lombalgia entre Fisioterapeutas que atuam em Santarém - PA. A amostra foi composta por 21 fisioterapeutas e, para coleta dos dados, utilizou-se um formulário on-line baseado em questionários existentes, sendo eles: Oswestry, Critério Macnab, Roland Morris e Questionário Nórdico, associado a escala EVA. Os dados obtidos foram analisados através da estatística descritiva quantitativa. Verificou-se na pesquisa a presença de um alto índice de dor, dormência e desconforto na região lombar. A amostra apresentou idade média de 29 anos, sendo que o gênero feminino teve um índice maior, e segundo Farinha et al. (2013), a idade média ficou entre 26 e 30 anos. Apesar de um índice maior relatado pelos participantes nos últimos 12 meses, os mesmos relataram que essas dores não interferem nas suas atividades laborais normais. E, em geral, a dor relatada pela escala EVA foi moderada. Diante da dimensão dos dados encontrados, sugere-se que mais pesquisas relacionadas a esse tema sejam realizadas para aprofundamento do estudo na área.
\end{abstract}

Palavras-chave: Lombalgia; Fisioterapeutas; Ergonomia; Saúde do trabalhador.

\begin{abstract}
Low back pain is a pathology that affects several groups of workers worldwide, being one of the most common musculoskeletal disorders today. It can interfere with the biomechanics of the entire spinal segment, leading to movement restriction and decreased muscle strength, which reflects on absences from work and, consequently, on the quality of life of these individuals. This study sought to analyze the profile of the incidence of low back pain among Physiotherapists working in Santarém - PA. The sample consisted of 21 physical therapists and, for data collection, an online form based on existing questionnaires was used, namely: Oswestry, Macnab Criterion, Roland Morris and Nordic Questionnaire, associated with the VAS scale. The data obtained were through quantitative descriptive statistics. It was found in the research the presence of a high rate of pain, numbness and discomfort in the lumbar region. The sample had an average age of 29 years, and females had a higher rate, and according to Farinha et al. (2013), the average age was between 26 and 30 years old. Despite a higher rate reported by participants in the last 12 months, they reported that these pains do not interfere with their normal work activities. And, in general, the pain reported by the VAS scale was moderate. Given the size of the data found, it was found that more research related to this theme is carried out to deepen the study in the area.
\end{abstract}

Keywords: Low back pain; Physiotherapists; Ergonomics; Worker's health. 


\begin{abstract}
Resumen
La lumbalgia es una patología que afecta a varios grupos de trabajadores a nivel mundial, siendo uno de los trastornos musculoesqueléticos más comunes en la actualidad. Puede interferir con la biomecánica de todo el segmento espinal, provocando restricción de movimiento y disminución de la fuerza muscular, lo que se refleja en las ausencias del trabajo y, en consecuencia, en la calidad de vida de estos individuos. Este estudio buscó analizar el perfil de la incidencia de lumbalgia entre los fisioterapeutas que trabajan en Santarém - PA. La muestra estuvo formada por 21 fisioterapeutas y, para la recogida de datos, se utilizó un formulario online basado en cuestionarios existentes, a saber: Oswestry, Macnab Criterio, Roland Morris y Nordic Questionnaire, asociado a la escala EVA. Los datos obtenidos fueron mediante estadística descriptiva cuantitativa. Se encontró en la investigación la presencia de una alta tasa de dolor, entumecimiento y malestar en la región lumbar. La muestra tenía una edad promedio de 29 años y las mujeres tenían una tasa más alta, y según Farinha et al. (2013), la edad promedio fue entre 26 y 30 años. A pesar de una tasa más alta informada por los participantes en los últimos 12 meses, informaron que estos dolores no interfieren con sus actividades laborales normales. Y, en general, el dolor informado por la escala EVA fue moderado. Dado el tamaño de los datos encontrados, se encontró que se realizan más investigaciones relacionadas con este tema para profundizar el estudio en el área.
\end{abstract}

Palabras clave: Lumbalgia; Fisioterapeutas; Ergonomía; Salud del trabajador.

\title{
1. Introdução
}

Segundo Silva e Silva (2005), a dor lombar é uma patologia que afeta diversos grupos de trabalhadores em âmbito mundial e, de forma geral, é uma das afecções musculoesqueléticas mais comuns na sociedade moderna. De acordo com Siqueira et al. (2008), pode interferir na biomecânica de todo o segmento da coluna, levando a restrição de movimento e diminuição da força muscular, o que reflete em afastamentos das atividades profissionais e diminuição na percepção da qualidade de vida desses indivíduos. Ou seja, a queixa álgica lombar pode ser considerada como um problema de saúde pública mundial.

Conceituando a lombalgia, de acordo com Pires e Samulski (2006), pode ser classificada como uma dor aguda, quando durar em torno de seis semanas; subaguda, com duração de até doze semanas; e, crônica, caso ultrapasse doze semanas. Alguns fatores que trazem cronicidade são: problemas psicológicos, trabalhos pesados com posturas em pé ou sentado, sedentarismo, horas excessivas de trabalhos, entre outros.

De acordo com a Organização Mundial da Saúde (1998), a qualidade de vida pode ser definida como o indivíduo percebe sua posição na vida, levando em consideração o contexto de cada cultura e valores nos quais está inserido e, também, em relação aos seus objetivos, expectativas, padrões e preocupações. E de acordo com França et al. (2015), um dos principais fatores que pode afetar a qualidade de vida, é a queixa álgica na região lombar, o que pode levar a limitação da capacidade de realização de suas atividades de vida diária e trabalho, pode também afetar aspectos de bem-estar, psicológico e qualidade do sono.

Aprendemos no decorrer da formação acadêmica que o Fisioterapeuta é o profissional de primeiro contato, e possui um amplo campo de atividade, desde a atenção primária, passando pela ambulatorial até a atenção terciária, atuando na terapia intensiva. Isso significa que, por muitas vezes, esse profissional trabalha em mais de uma área de atendimento, ou seja, aumentando sua exposição a distúrbios osteomioarticulares pela sobrecarga.

O objetivo do trabalho foi realizar o levantamento de ocorrências de dor lombar entre o grupo de Fisioterapeutas atuantes da cidade de Santarém-PA. De acordo com o levantamento do COFFITO, no ano de 2018, existiam no Brasil cerca de 240 mil Fisioterapeutas (COFFITO, 2021), e na cidade de Santarém encontramos um grande grupo desses profissionais em atuação, e muitas instituições de formação de futuros profissionais. Verificar a incidência de dor lombar entre os profissionais de nossa cidade demonstra nossa preocupação, ainda enquanto acadêmicos, em relação à qualidade de vida desses indivíduos, e também em fomentar a discussão e levantar dados que subsidiem pesquisas e intervenções de futuros pesquisadores.

No contexto pandêmico dos últimos dois anos, foi possível verificarmos o aumento da procura pelos serviços de fisioterapia, e, devido a isso, a carga de trabalho desses profissionais se elevou substancialmente, o que ainda mais nos levou a questionar o índice de lombalgia entre esse público. 


\section{Metodologia}

O presente estudo caracteriza-se como estudo exploratório, analítico e descritivo com uma abordagem quantitativa. A pesquisa ocorreu nas clínicas ambulatoriais de Fisioterapia da cidade de Santarém, onde a média de atendimento era de 8 horas diárias. A amostra foi composta por 21 Fisioterapeutas de ambos os gêneros, que atuavam como prestadores de serviços ou carteira assinada nessas clínicas. Todos concordaram em participar da pesquisa por meio da assinatura do Termo de Consentimento Livre e Esclarecido (TCLE). Como critérios de inclusão, levou-se em consideração Fisioterapeutas prestadores de serviços e/ou carteira assinada; de ambos os gêneros; com idade adulta entre 19 a 59 anos, e que atuassem no mínimo 20 horas semanais. E como critérios de exclusão, não participaram profissionais que não consentiram em assinar o Termo de Consentimento Livre e Esclarecido; profissionais que prestavam serviços esporádicos, de forma particular. e sem horário ou sessões fixas; profissionais afastados por critérios de saúde mental ou física, que os impossibilitassem de utilizar o questionário desta pesquisa, ou que não foram encontrados para responder os questionários após 5 tentativas de contato.

Foi utilizado, como instrumento de coleta de dados, um questionário baseado nos estudos de Roland e Morris (1983), denominado de Questionário de Incapacidade de Roland e Morris, adaptado para o português por Nusbaum et al. (2001). O mesmo instrumento foi baseado no trabalho de Kuorinka et. al (1987), na versão em inglês "Nordic Musculoskeletal Questionnaire - NMQ), o qual Barros e Alexandre (2003) adaptaram para a versão brasileira, denominado "Questionário Nórdico de Sintomas Osteomusculares - QNSO). O questionário foi baseado, ainda, nos trabalhos de Fairbank et al. (1980), com o Questionário de deficiência de dor lombar - Oswestry, e na Escala Visual Analógica da dor, apresentado por Falavigna et al (2011). A Escala Visual Analógica da dor consistia em um escore numérico de aferição da intensidade da dor pelo paciente, apresentado em uma linha reta que variava de 0 a 10, sendo zero ausência de dor e 10 a pior dor. Como suporte metodológico utilizamos a obra de Estrela, C. (2018), Metodologia Científica: Ciência, Ensino, Pesquisa. Editora Artes Médicas.

A pesquisa foi aprovada pelo CEP Centro Universitário Maurício de Nassau, sob o número de protocolo: 4.956.124. O procedimento de campo deu-se da seguinte forma: o primeiro contato com as clínicas foi para apresentar a pesquisa e o TCLE, e pedir a autorização, por meio de ofício, para formalizar a autorização dos responsáveis. Após isso, os Fisioterapeutas tiveram a oportunidade de ler, tirar as dúvidas em relação à pesquisa e assinarem o termo, caso estivessem de acordo em participar. Logo após, foi enviado o Questionário, de forma on-line, para os representantes das clínicas, para que reenviassem para os seus Fisioterapeutas.

\section{Resultados e Discussão}

Esta pesquisa contou com uma amostra de 21 fisioterapeutas que atuavam em 5 clínicas particulares na cidade de Santarém. Desses, 13 eram mulheres (61,9\%) e 8 eram homens (38,1\%); em relação a tempo de atividade, 11 deles trabalham mais de 3 anos (52,4\%), 4 indivíduos trabalham de 1 a 2 anos (19\%) e 6 deles menos de 1 ano (28,6\%). A média de trabalho semanal foi de 47 horas semanais. Constatou-se, então, que da amostra, a maioria dos Fisioterapeutas trabalham em mais de um local, seja como docente, área hospitalar, neuro funcional, e alguns deles também são proprietários de clínica.

Em relação a amostra, 17 Fisioterapeutas relataram sentir dor, dormência e desconforto na região lombar, isso representa $81 \%$ da amostra; além disso, 4 alegaram não sentir dor (19\%), conforme o gráfico 1. Respondendo ainda em relação a porcentagem de Fisioterapeutas que sentiram alguns desses sintomas, ao comparar-se com os achados no trabalho de Pivetta et al. (2005), onde verificou-se que 90,7\% deles referiram sentir algum distúrbio osteomuscular em alguma região do corpo, e em relação a região lombar, esse número chegou a 51,16\%, ou seja, pouco mais da metade dos Fisioterapeutas entrevistados sentiram algum dos sintomas acima citados. No estudo de Gama (2012), a porcentagem de dor lombar chegou a 60\%. Já na pesquisa de Vieira et al. (2016), 66\% da amostra sentiu dor e desconforto lombar. Vieira et al. (2015) verificou, que da sua amostra, 67,5\% sentiu dor lombar. Já no estudo de Alexopoulos et al (2011) foram 51\% dos indivíduos os que sentiram dor lombar. Já a 
investigação de Pivetta et al. (2005) apresentou 51,16\% de incidência de dor lombar. No estudo de Siqueira et al. (2008), que identificou um alto índice de distúrbios osteomusculares na região lombar, correspondendo a 78,58\% de queixas.

Nesta pesquisa, a média de idade é de 29 anos, já no trabalho de Pivetta et al. (2005), a idade ficou entre 21 e 30 anos, que se configurou como a idade média dos fisioterapeutas afetados por algum distúrbio osteomuscular na região lombar, corroborando, ainda, com Carregaro et al. (2005), que em sua pesquisa demonstrou que a idade média ficou entre 20 e 25 anos. No estudo de Islam et al. (2015), a idade média dos fisioterapeutas foi de 28 anos. Verificou-se, também, que dos 21 Fisioterapeutas da amostra, 9 indivíduos (42,9\%) sentiram alguns dos sintomas, e trabalhavam a mais de 3 anos como Fisioterapeutas. No estudo de Siqueira et al. (2008), verificou-se que a média ficou em 8 anos de trabalho.

Gráfico 1: Relato de dor ou desconforto de fisioterapeutas nos últimos 12 meses (n=21).

\section{INDIVÍDUOS QUE RELATARAM SENTIR DOR NOS ÚLTIMOS 12 MESES}

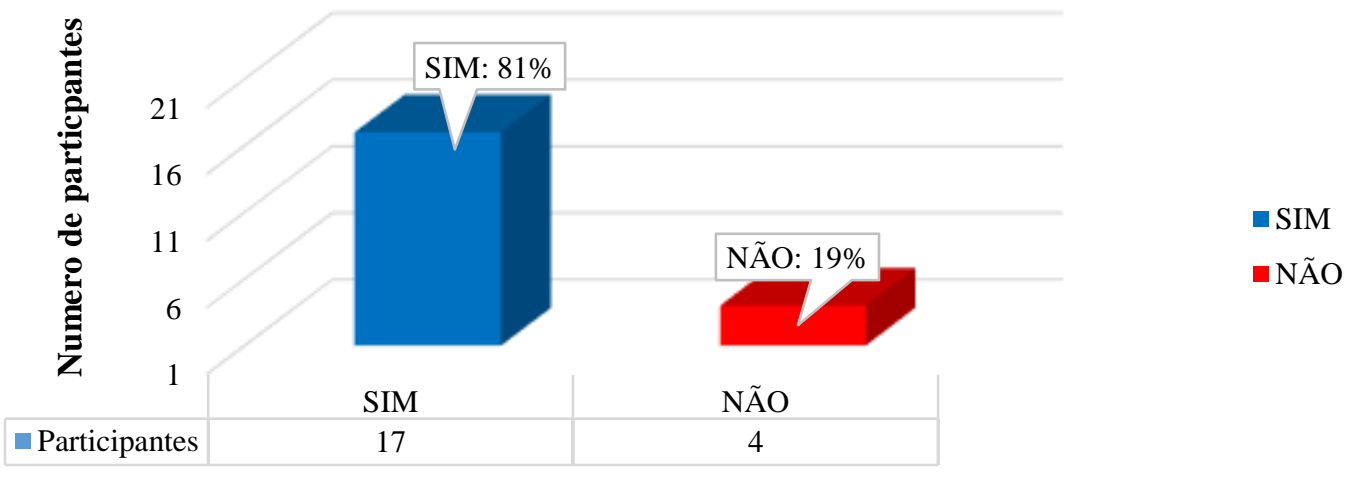

\section{Respostas dos entrevistados}

Fonte: Dados da pesquisa (2021).

Em relação ao questionamento se nos últimos 12 meses tiveram que evitar atividades normais, como resposta foi notado que por causa de problema na região lombar, constatou-se que: 16 relataram que não (76,2\%) e 5 disseram que sim (23,8\%). Ao comparar com o estudo de Silva et al. (2014), mesmo sendo baseado em pesquisa com Fisioterapeutas e enfermeiros no âmbito hospitalar, verificou-se que $11,6 \%$ da amostra relatou ter que se afastar das suas atividades normais por alterações na região lombar. Já nos últimos 7 dias, 9 indivíduos relataram que sentiram alguma dor, desconforto ou dormência (42,9\%) e 12 disseram que não $(57,1 \%)$ conforme o Gráfico 2. No estudo de Silva et al. (2014), 25,6\% deles foram acometidos por algum desconforto na lombar. Outro ponto importante é a influência da dor lombar com o humor, 9 relataram ficar mais irritado ou mal humorado com as pessoas do que o habitual $(42,9 \%)$, e 12 relataram que não $(57,1 \%)$. 
Gráfico 2: Relato de dor e desconforto de fisioterapeutas nos últimos 7 dias $(n=21)$

INDIVÍDUOS QUE SENTIRAM DORES NOS ÚLTIMOS 7 DIAS

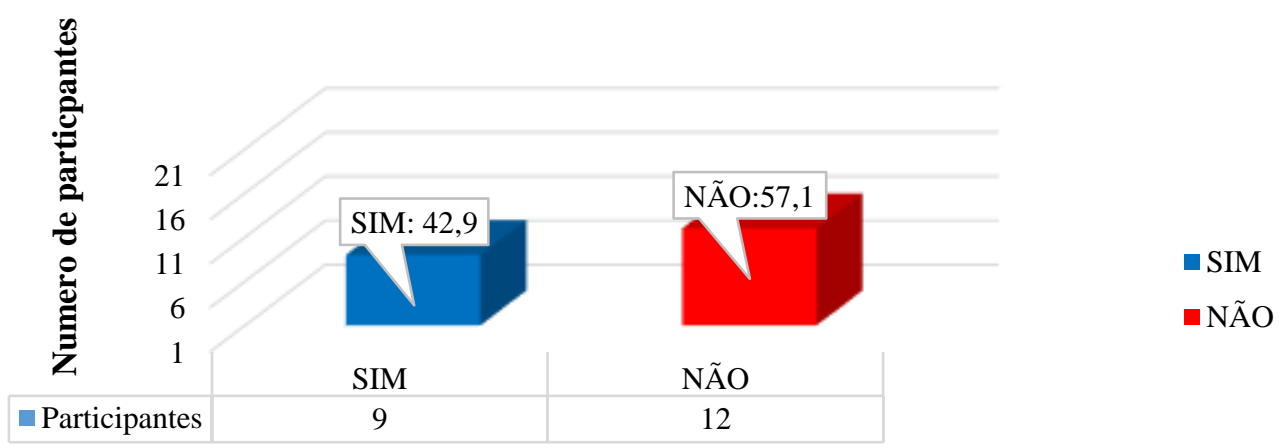

Respostas dos entrevistados

Fonte: Dados da pesquisa (2021).

Ressalta-se ainda a escassez em relação a artigos e pesquisas voltadas para essa área, e os poucos que foram encontrados são muito antigos. Não foi encontrado nenhum artigo que correlacionasse a influência da dor lombar com o humor dos participantes.

Em relação ao resultado da escala EVA, percebeu-se que 8 indivíduos relataram média de 4, isso representa 38,1\%, 3 indivíduos marcaram média 5, o que representa 14,3\%, 2 (9,5\%) marcaram o índice 7, 1 (4,8\%) marcou o índice 6, ambos representam dor moderada segundo a escala; 3 indivíduos (14,3\%) marcaram o índice 2, dor leve, e 4 indivíduos (19\%) relataram não sentir dor alguma, como podemos perceber no gráfico 3 abaixo. De acordo com Siqueira et al. (2008), utilizando a Escala visual numérica, outro termo para Escala visual analógica da dor, demonstrou que 59,02\% dos indivíduos sentiram dor localizada, $13,11 \%$ dor em queimação, 24,59\% dor que irradiava para os MMII e 3,28\% dor latejante.

Gráfico 3: Escala analógica da dor $(n=21)$.

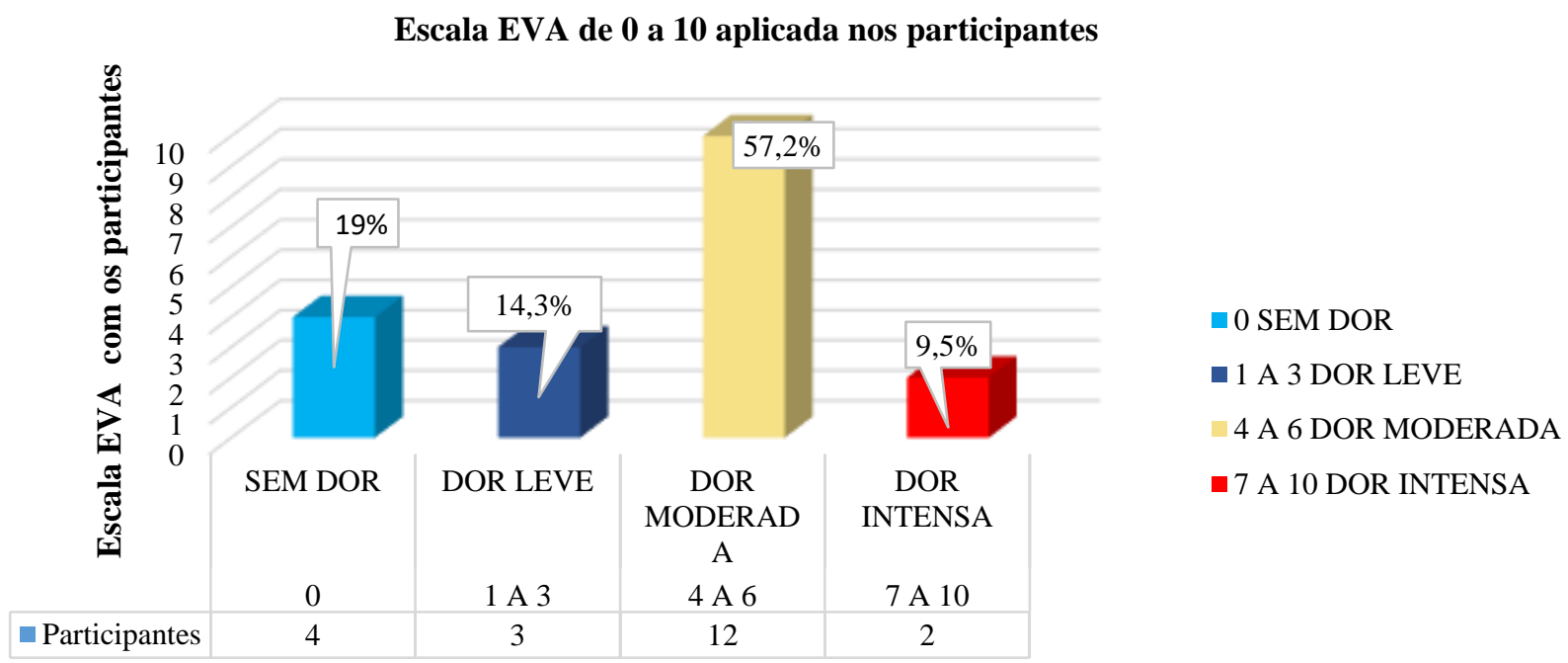

Respostas dos entrevistados

Fonte: Dados da pesquisa (2021). 
Outro achado importante na pesquisa foi em relação ao relato de queixas de dores lombares relacionada ao gênero, obteve-se ainda nos resultados que as mulheres tiveram um índice maior que os homens, onde 47,5\% eram do sexo feminino e 33,5\% eram do sexo masculino. Porém, ressalta-se que 4 indivíduos da amostra não sentiram dor lombar (19\%), de acordo com o Gráfico 4. Como resposta foi notado que a maior prevalência então foi entre o sexo feminino. Corroborando com o estudo de Siqueira et al. (2008), onde 98,2\% das queixas vinham do sexo feminino. O que demonstra um alto índice de dor, desconforto lombar em fisioterapeutas do sexo feminino. No estudo de Santos et al. (2018), 83\% eram do sexo feminino, e de acordo com Almeida et al. (2008), as mulheres mostraram-se mais suscetíveis a este distúrbio, em relação aos homens.

Gráfico 4: Dor lombar em relação ao gênero Masculino x Feminino.

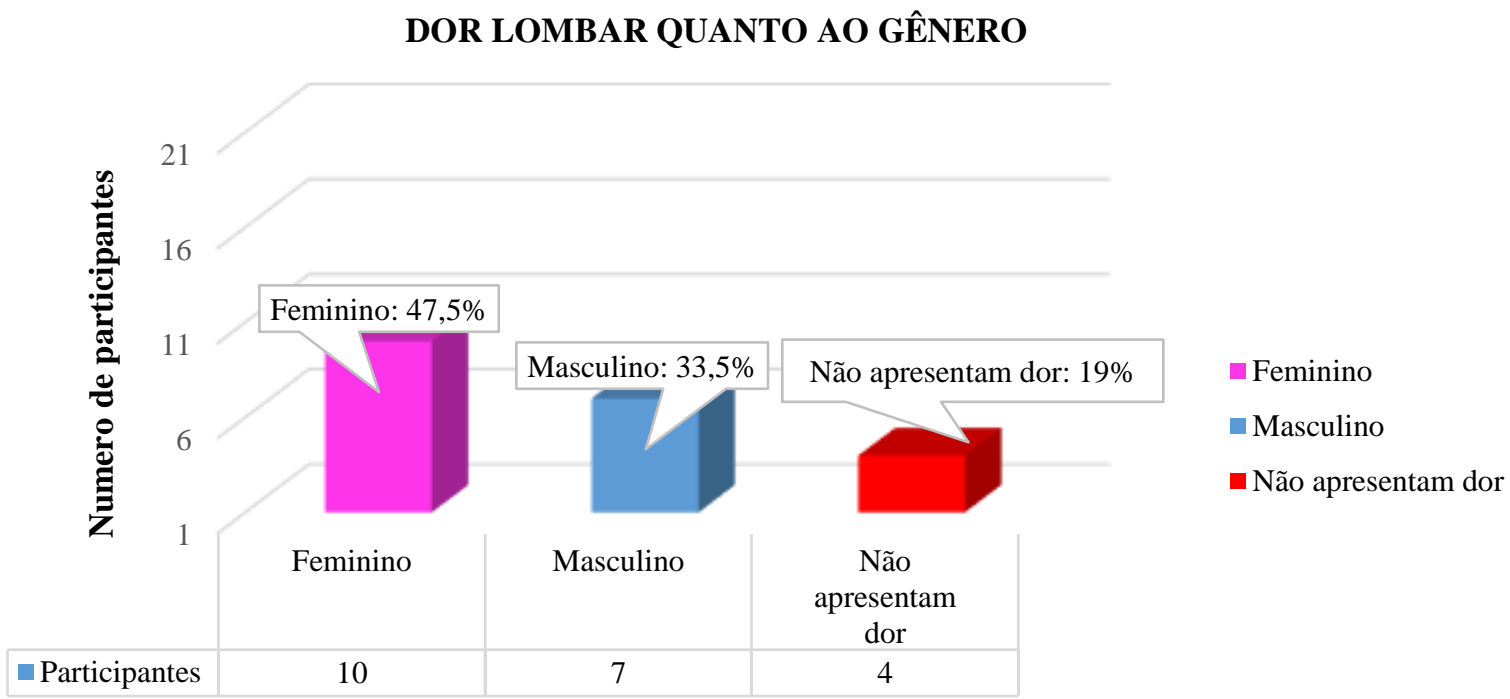

\section{Respostas dos entrevistados}

Fonte: Dados da pesquisa (2021).

\section{Conclusão}

Esta pesquisa encontrou um alto índice de relatos de distúrbios na região lombar, como dor moderada, desconforto e dormência. A intensidade da dor entre os participantes ficou entre 4 e 5 no índice da Escala EVA, o que se caracteriza como dor moderada. Sendo que o gênero mais afetado é o sexo feminino. No entanto, outro ponto a ser destacado é como essa dor influencia no mau humor desses indivíduos, pois um número significante da amostra relatou que a dor influencia para contribuir com o mau humor deles.

Em relação a idade média dos participantes, foi de adultos jovens, em torno de 30 anos, o que preocupa devido a precocidade dos aparecimentos dos distúrbios. No entanto, devido ao número limitado da amostra e a escassez de estudos relacionados ao perfil de dores lombares em Fisioterapeutas, sugere-se o desenvolvimento de trabalhos futuros referentes a este tema, especificamente como o distúrbio surge, em quais áreas da Fisioterapia os índices são maiores e o quê os próprios Fisioterapeutas podem fazer para diminuir esse quadro, pois isso reflete diretamente a qualidade de vida desses profissionais, e isso resulta num melhor esclarecimento e comparação dos resultados. 


\section{Referências}

Alexopoulos E., Tanagra D., Detorakis I., Gatsi P., Goroyia A., \& Michalopoulou M. (2011). Knee and low back complaints in professional hospital nurses: occurrence, chronicity, care seeking and absenteeism, Work, 38(4), 329-335.

Almeida, F. R. T. D., Brandão, B. B., \& Rocha, C. A. Q. (2008). Índice de lesões e afecções musculoesqueléticas relacionadas aos profissionais da área de fisioterapia da cidade de Muriaé, MG. Revista Científica da Faminas ,4(3).

Barros, E. N. C., \& Alexandre, N. M. C. (2003). Cross-cultural adaptation of the Nordic musculoskeletal questionnaire. International nursing review. Jun;50(2): $101-8$.

Carregaro, R. L., Trelha, C. S., \& Mastelari, H. J. Z. (2006). Distúrbios osteomusculares relacionados ao trabalho em fisioterapeutas: revisão da literatura. Fisioterapia e Pesquisa, 13(1), 53-59.

Conselho Federal de Fisioterapia e Terapia Ocupacional. (07 de setembro de 2021). Fisioterapia: definições e área de atuação. http://www.coffito.org.br/publicacoes/pub_view.asp?cod=1007\&psecao=9.

Estrela, C. (2018). Metodologia Científica: Ciência, Ensino, Pesquisa. Editora Artes Médicas. (3ª edição).

Falavigna, A., Teles, R. A., Braga, G. L., Barazzettti, D. O., Lazzaretti, L., \& Tregnago, A. C. (2011). Instrumentos de Avaliação clinica e funcional em cirurgia da coluna vertebral. Coluna/Columna,10 (1): 62-7.

Fairbank, J. C., Couper, J., Davies, J. B., \& O’brien, J. P. (1980). The Oswestry low back pain disability questionnaire. Physiotherapy, 66(8), $271-273$.

Farinha, K. O., Almeida, M. S., \& Trippo, K. V. (2013). Avaliação da qualidade de vida de docentes fisioterapeutas da cidade do Salvador/ Bahia. Revista Pesquisa em Fisioterapia, 3(1).

França, V. L., Koerich, M. H. A. D. L., \& Nunes, G. S. (2015). Sleep quality in patients with chronic low back pain. Fisioterapia em Movimento, $28,803-810$.

Gama, K. C. S. D. (2012). Avaliação álgica em profissionais de fisioterapia da área de Traumato-ortopedia em Vitória da Conquista - BA. Ciência \& Desenvolvimento-Revista Eletrônica da FAINOR, 5(1).

Islam, S., Monjurul, H., Abdul, H., \& Debra, L, Rahman (2015). Musculoskeletal complaints among physiotherapy and occupational therapy rehabilitation professionals in Bangladesh. Work, 50(3), 379-386.

Kuorinka, I., Jonsson, B., Kilbom, A., Vinterberg, H., Biering-Sørensen, F., Andersson, G., \& Jørgensen, K. (1987). Standardised Nordic questionnaires for the analysis of musculoskeletal symptoms. Applied ergonomics, 18(3), 233-237.

Nusbaum, L., Natour, J., Ferraz, M. B., \& Goldenberg, J. (2001). Translation, adaptation an validation of the Roland-Morris quesrtionnaire - Brazil RolandMorris. Brazilian Journal of Medical and Biological Research, 34(2), 203-210.

Organização Mundial da Saúde. (1998). Promoción de la salud: glosario (No. WHO/HPR/HEP/98.1). Genebra: Organización Mundial de la Salud.

Pires, F. D. O., \& Samulski, D. M. (2006). Visão interdisciplinar na lombalgia crônica causada por tensão muscular. Revista Brasileira de Ciência e movimento, 14(1), 13-20.

Pivetta, A. D., Jacques, M. A., Agne, J. E., \& Lopes, L. F. (2005). Prevalência de distúrbios osteomusculares relacionados ao trabalho em fisioterapeutas. Revista Digital: Buenos Aires, 10(80).

Santos, R. M. E. D., Maduro, P. A., Da Silva, T. F. A., \& Trombini-Souza, F. (2018). Dor e desconforto musculoesquelético em fisioterapeutas da unidade de terapia intensiva e enfermaria de um hospital universitário: um estudo de coorte retrospectivo. BrJP, 1, 127-133.

Silva, C. B. D., Rocha, C. S. A., Kawano, M. M., Neto, M. G., \& Martinez, B. P. (2014). Sintomas Osteomusculares em Fisioterapeutas e Enfermeiros no ambiente hospitalar. Revista Pesquisa em Fisioterapia, 4(3).

Silva, C. D. S., \& Silva, M. A. G. (2005). Lombalgia em fisioterapeutas e estudantes de fisioterapia: um estudo sobre a distribuição da frequência. Fisioterapia Brasil, 6(5), 376-380.

Siqueira, G. R., Cahu, F. G. M., \& Vieira, R. A. G. (2015). Ocorrência de lombalgias em fisioterapeutas na cidade de Recife, Pernambuco. Brazilian Journal of Physical Therapy, 12, 222-227.

Vieira, E. R., Svoboda S., Belniak, A., Brunt, D., Prix, C. R. St., Roberts, L., \& Costa, B. R. D. (2016). Work related musculoskeletal disorders among physical therapists: an online survey. Disability and rehabilitation, 38(6), 552-557.

Vieira, W. H., Pedroza E. P., Filho R. G., \& Abreu B. J. (2015). Prevalência de desconforto musculoesquelético em fisioterapeutas da rede pública hospitalar de Natal/RN. Fisioterapia Brasil, 16(2), 107-112. 